\title{
Highly sensitive detection of human thrombin in serum by affinity capillary electrophoresis/laser-induced fluorescence polarization using aptamers as probes
}

\author{
Maoyong Song, Yuexia Zhang, Tao Li, Zhixin Wang, Junfa Yin, Hailin Wang* \\ State Key Laboratory of Environmental Chemistry and Ecotoxicology, Research Center for Eco-Environmental Sciences, Chinese Academy of Sciences, \\ Beijing 100085, China
}

\section{A R T I C L E I N F O}

\section{Article history:}

Received 29 October 2008

Received in revised form

25 November 2008

Accepted 27 November 2008

Available online 6 December 2008

\section{Keywords:}

Affinity probe capillary electrophoresis

Laser-induced fluorescence polarization

Aptamer

Thrombin

\begin{abstract}
A B S T R A C T
The detection and quantification of disease-related proteins play critical roles in clinical practice and diagnostic assays. We present an affinity probe capillary electrophoresis/laser-induced fluorescence polarization (APCE/LIFP) assay for detection of human thrombin using a specific aptamer as probe. In the APCE/LIFP assay, the mobility and fluorescence polarization of complex are measured simultaneously during CE analysis. The affinity complex of human thrombin can be well separated from unbound aptamer on CE and clearly identified on the basis of its fluorescence polarization and migration. Because of the binding favorable G-quartet conformation potentially involved in the specific aptamer, it was assumed that monovalent and bivalent cations promoting the formation of a stable $G$ quadruplex conformation in the aptamer may enhance the binding of the aptamer and thrombin. Therefore, we investigated the effects of various metal cations on the binding of human thrombin and the aptamer. Our results show that cations like $\mathrm{K}^{+}$and $\mathrm{Mg}^{2+}$ could not stabilize the affinity complex. Without the use of typical cations, a highly sensitive assay of human thrombin was developed with the corresponding detection limits of $4.38 \times 10^{-19}$ and $2.94 \times 10^{-19} \mathrm{~mol}$ in mass for standard solution and human serum, respectively.
\end{abstract}

(c) 2008 Elsevier B.V. All rights reserved.

\section{Introduction}

Thrombin is a $\mathrm{Na}^{+}$-activated allosteric serine protease that converts soluble fibrinogen into insoluble fibrin, as well as catalyzing many other coagulation-related reactions in the blood coagulation cascade [1]. As a multifunctional protein, thrombin gives rise to factors XI, V, and VII, and activates protein $\mathrm{C}$ which acts as an inhibitor of the coagulation cascade [2,3], and promotes platelet activation through protease-activated receptor [4]. Activation of prothrombin plays a crucial role in physiological and pathological coagulation. Various diseases involving prothrombin and thrombin have been reported, such as Alzheimer's disease [5] and thromboembolic disease [6]. On the other hand, the US Food and Drug Administration approved human thrombin to be used to help control bleeding during surgery [7]. Thus, it is important and necessary to detect thrombin in blood not only for patients suffering from diseases known to be associated with coagulation abnormalities [8], but also for determining the effectiveness of therapeutic drugs after surgery or in thromboembolic disease treatment $[9,10]$.

\footnotetext{
* Corresponding author. Tel.: +86 10 62849600; fax: +86 1062849600 .

E-mail address: hlwang@rcees.ac.cn (H. Wang).
}

Thrombin is not present in blood under normal conditions. The concentration of thrombin in blood can vary over a large range, from picomoles/liter to micromoles/liter during the coagulation process $[11,12]$. Thus, the sensitivity of detection and quantification of thrombin plays essential role in clinical practice as well as in diagnostic assays. The thrombin in blood can be determined by several methods including clotting-based assays [13], synthetic substrate-based enzymatic activity assays [14,15], and immunoassays [16]. But none of these tests is adequately sensitive or specific [17]. Recently, a novel affinity probe, aptamer has been employed to measure thrombin using APCE $[18,19]$.

Aptamers are single-stranded DNA or RNA oligonucleotides selected by systematic evolution of ligands by exponential enrichments (SELEX) based on their ability to bind target biomolecules with high affinity and specificity $[20,21]$. Aptamers possess some advantageous properties over antibodies, such as ease-of-synthesis, ease-of-labeling and excellent stability. Alternatives to antibodies, aptamers have been pursued as affinity probes or ligands for protein detection in several methods [22-24]. It is also likely to act as a class of molecules that rival antibodies in both therapeutic and diagnostic applications [25]. There are two anti-thrombin aptamers with high affinity. One is a 15-mer single-stranded DNA which forms an intramolecular quadruplex structure and binds to thrombin at the fibrinogen-recognition exosite [26,27]. Another 29-mer one has 
been proved to bind at heparin-binding exosite with a higher affinity [28]. When these aptamers are used in APCE/LIF, the assay has showed potential properties of smaller sample size, high sensitivity, rapid separation, and compatibility with automation [29] for thrombin detection.

The objective of this work is to develop an aptamer-based method for detection of thrombin at sub-nM levels in complex matrices. To achieve this objective, a method of APCE/LIFP, which allows for on-line monitoring of affinity complex formation, has been used. The theory of fluorescence polarization (FP), first described in 1926 by Perrin [30], is based on the molecular movement and rotation. Briefly, the theory is based on the fact that molecules can naturally rotate in a liquid medium. The rate of molecular rotation is a function of the molecule's size. Larger molecules rotate at a slower rate than smaller molecules do. A fluorescent dye attached to a specific molecule can be used to quantitatively evaluate the change in fluorescence polarization of the tagged molecule associated with the change of the molecule's size, which could be attributed to the binding of tagged ligand and target receptor. If the fluorescent dye tagged molecule (aptamer) binds to the target molecule (thrombin), the formation of the aptamer-thrombin complex creates a larger size that makes it to rotate at a slower rate and produces a larger polarization value. Fluorescence polarization is hence used to identify and detect the binding of aptamer to thrombin. When coupled with $\mathrm{CE} / \mathrm{LIF}$, the mobility and fluorescence polarization of the complex between fluorescence labeled aptamer and thrombin can be monitored simultaneously, thereby providing more useful information on binding reactions. This CE/LIFP method has been successfully used to the study protein-peptide [31], protein-protein [32] and protein-DNA [33] interactions in previous work.

In this report, we applied CE/LIFP to highly sensitive detection of thrombin in standard solution and in spiked human serum using a fluorescently labeled aptamer as a specific probe for human thrombin. Unbound aptamer and aptamer-thrombin complex were well separated on CE, and the migration and fluorescence polarization of the complex were measured simultaneously. Despite the reported enhancement of thrombin-aptamer binding by some metal cations, the measured dissociation constant $\left(k_{d}\right)$, dissociation rate constant $\left(k_{o f f}\right)$, and the fluorescence polarization of the thrombin-aptamer complex consistently indicates that the presence of metal cations (e.g. $\mathrm{K}^{+}$and $\mathrm{Mg}^{2+}$ ) are not favorable in formation of complex. By using optimized aptamer based on CE/LIFP analysis, the limit of detection (LOD) for thrombin was $4.38 \times 10^{-19}$ and $2.94 \times 10^{-19} \mathrm{~mol}$ $(S / N=3)$ in mass (83 and $55.6 \mathrm{pM}$ in concentration) for standard solution and human serum, respectively. This sensitive CE/LIFP method can be extended to other protein detection using high affinity aptamer probes.

\section{Materials and methods}

\subsection{Chemicals and reagents}

All solutions were prepared with $18.2 \mathrm{M} \Omega$ distilled deionized water (ALGA system, UK). TG buffer $(1 \times, 25 \mathrm{mM}$ Tris, $192 \mathrm{mM}$ glycine, $\mathrm{pH}$ 8.3) was prepared with the chemicals of analytical pure grade, obtained from commercial sources, and filtered with a $0.22-\mu \mathrm{m}$ filter (Millipore, Bedford, MA, USA). Two tetramethylrhodamine (TMR) labeled aptamers, TA29 (5'-TMRAGT CCG TGG TAG GGC AGG TTG GGG TGA CT) and TA35 (5'-AAT TTT AGT CCG TGG TAG GGC AGG TTG GGG TGA CT-TMR-3'), were synthesized by Sangon Biological Engineering Technology and Services (Shanghai, China). Bovine serum albumin (BSA, 66 kDa) was purchased from Sigma (St. Louis, MO, USA). Human serum was obtained from healthy adults.

\subsection{Instrument}

The aptamer-based APCE analysis was conducted on a laboratory-built CE/LIFP system which consisted of a high-voltage power supply (Model DW-S303-1ACE5/DW-HVSC-1, Tianjin Dongwen High-voltage Power Supply Plant, Tianjin, China), a fused-silica capillary, laser and a laser-induced fluorescence (LIF) detector. The TMR labeled aptamers were excited with a $543.5 \mathrm{~nm}$ helium-neon laser of $1 \mathrm{~mW}$ (Melles Griot, Irvine, CA, USA). The emission was focused by a $60 \times$ microscope objective (Shanghai Cany Precision Instrument Co., Shanghai, China) at the perpendicular direction to the laser beam. Fluorescence was passed through a pinhole, and filtered through a FF01-575/15-25 filter (Semrock, Rochester, NY, USA). A polarizing beam splitter (Melles Griot, Nepean, Canada) was used to split the fluorescence beam into horizontally and vertically polarized light beams, which could be measured by two photomultiplier tubes (PMT, Model R1477, Hamamatsu Photonics, Japan), respectively. A current-to-voltage converter transferred the current signal from the PMT to voltage signal, which could be acquisted by a Lenovo personal computer using a HW-2000 chromatographic work station (Qianpu Software, Shanghai, China).

\subsection{Thrombin-aptamer reaction}

Aptamer stock solution was stored at $-20^{\circ} \mathrm{C}$, and thrombin stock solution was stored at $-80^{\circ} \mathrm{C}$. Before sample preparation, the stock solution of aptamer was reconstituted in TG buffer and heated at $95^{\circ} \mathrm{C}$ for $5 \mathrm{~min}$, then cooled on the ice. A certain volumes of thrombin and aptamer were mixed together, and diluted to the desired concentration with $1 \times$ TG buffer (final volume: about $20 \mu \mathrm{L}$ ). The mixture was vortexed for $30 \mathrm{~s}$ and incubated on ice for $30 \mathrm{~min}$ before injection to the capillary. The final concentration of the aptamer was kept as $5 \mathrm{nM}$ and that of thrombin was varied from 0 to $118 \mathrm{nM}$.

\subsection{CE/LIFP analysis}

Fused-silica capillary $(30 \mathrm{~cm} \times 25 \mu \mathrm{m}$ i.d.) used for separation was obtained from Yongnian Optic Fiber Plant (Hebei, China). A positive voltage of $15 \mathrm{kV}$ was applied for the electrokinetic injection, and $20 \mathrm{kV}$ for the CE separation, which carried out at room temperature. In order to reduce protein absorption on the inner capillary wall that may interfere with the reproducibility of the APCE measurement, the capillary was systematically flushed for $1 \mathrm{~min}$ with $0.02 \mathrm{M} \mathrm{NaOH}, 3 \mathrm{~min}$ with deionized water and then with running buffer for $5 \mathrm{~min}$ before each analysis. CE analysis of each sample was repeated three times. The fluorescence polarization (FP) values were calculated from the obtained intensity of the horizontally polarized fluorescence $\left(I_{h}\right)$ and vertically polarized fluorescence $\left(I_{v}\right)$ according to the following equation [34]:

$P=\frac{I_{v}-I_{h}}{I_{v}+I_{h}}$

\section{Results and discussion}

\subsection{Complex formation and CE separation}

A number of factors may affect the APCE/LIFP analysis of the thrombin-aptamer complex, such as buffer composition, CE voltage, and in particular capillary length. The success of affinity analysis of pre-incubated protein-aptamer solution depends upon the complex formation and subsequent stability during CE separation. Since long CE separation may increase the possibility of the dissociation of the thrombin-aptamer complex, a relatively short capillary (total length: $30 \mathrm{~cm}$, effective length: $23.5 \mathrm{~cm}$ ) was used to reduce $C E$ separation time and the dissociation of the complex. 


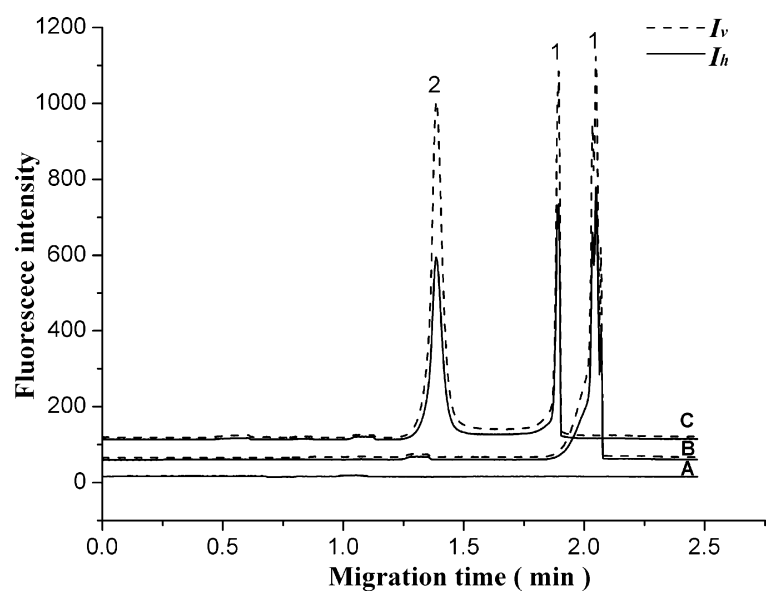

Fig. 1. Determination of thrombin-aptamer complex using APCE/LIFP analysis. (A) thrombin; (B) TA35; (C) thrombin + TA35. Peaks 1 and 2 represent the unbound aptamer TA35 and the complex of thrombin and TA35, respectively. Vertically $\left(I_{v}\right)$ and horizontally $\left(I_{h}\right)$ polarized fluorescence intensities are shown in electropherograms with dot and solid lines. The concentrations of thrombin and TA35 are 15 and $5 \mathrm{nM}$, respectively. The separation buffer and the sample buffer are $1 \times$ TG buffer (pH8.3) and $1 \times \mathrm{TG}$ buffer plus $10 \mathrm{mM} \mathrm{KCl}(\mathrm{pH} \mathrm{8.3)}$, respectively.

The TG buffer $(1 \times)$ was used as separation buffer. Fig. 1 shows the CE-LIFP analysis of $5 \mathrm{nM}$ aptamer with and without $15 \mathrm{nM}$ thrombin in the samples under such conditions. It is evident that the complex of thrombin-aptamer $(1.39 \mathrm{~min})$ is well separated from unbound aptamer (1.89 $\mathrm{min}$ ) (Fig. 1 trace $\mathrm{C})$. In the absence of thrombin, only the peak of aptamer is shown up in the electropherograms (Fig. 1 trace B). In this experiment, the horizontally polarized fluorescence $\left(I_{h}\right)$ and vertically polarized fluorescence $\left(I_{v}\right)$ were monitored simultaneously; therefore, the fluorescence polarization value could be derived. The complex has a higher $P$ (polarization) value $(P=0.304)$, and the unbound aptamer has a lower $P$ value $(P=0.196)$. The measured fluorescence polarization values of the bound and unbound aptamer probes are consistent with their size. In fact, CE/LIFP analysis not only provides the information on electrophoretic mobility (derived from migration), but also offers the information on the diffusional rotation of the analytes (fluorescence polarization). It has been demonstrated that the FP values can be used to differentiate between the unbound aptamers and the protein bound aptamer complexes even when they are not well separated $[35,36]$. Therefore, here we used the CE/LIFP approach to identify the thrombin-aptamer complex in CE separation of complex matrix.

To examine whether the protein thrombin and aptamer could form the complexes with different stoichiometry, a constant concentration of aptamer TA35 ( $5 \mathrm{nM}$ ) was titrated by different concentration of the thrombin protein (from 0.25 to $118 \mathrm{nM}$ ). For all titrated samples, CE-LIFP analysis only showed the two well-separated aptamer peaks, one for the complex of thrombin-aptamer and another for the unbound aptamer. The complex of thrombin-aptamer showed faster migration (1.39 min) and the bound aptamer showed slower migration (1.89 min) (Fig. 2). Furthermore, the peaks of the formed complex for all concentrations investigated were shown up with symmetry peak shape and no shoulder peak was observed. These results suggest that the binding of thrombin to aptamer TA35 only generates a sole complex with a stoichiometry of $1: 1$. To validate the sole binding stoichiometry of thrombin-aptamer TA35 complex (1:1), the fluorescence polarization responses of the complex formed in different concentrations were evaluated. The unbound aptamer has a low $P$ value $(P=0.196)$, and the bound aptamer has a markedly increased $P$ value $(P=0.304)$. The different fluorescence polarization can dis-

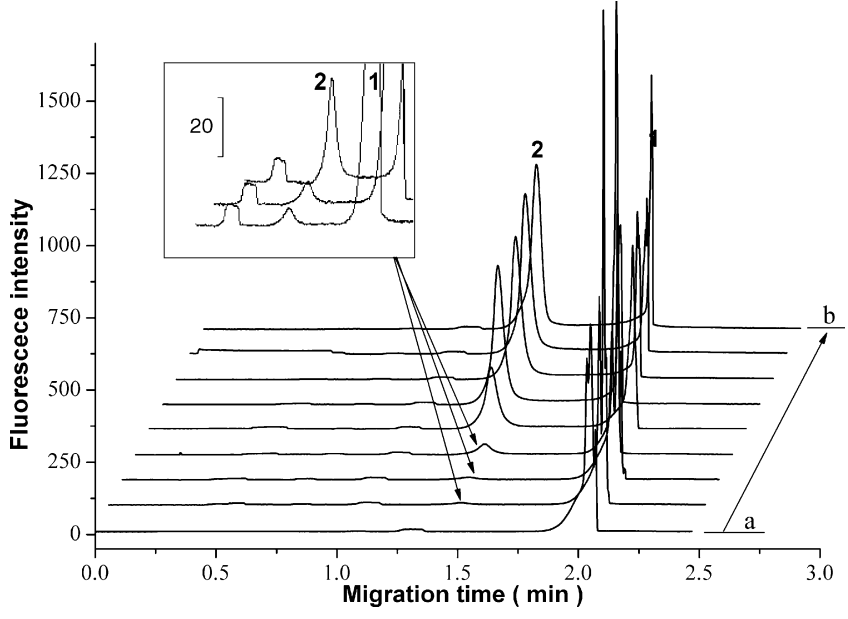

Fig. 2. CE/LIFP analysis of the mixture of aptamer and thrombin. The concentration of TA35 is $5 \mathrm{nM}$. The concentrations of thrombin (a to b) are $0,0.25,0.5,1,5,15,30$, 59 , and $118 \mathrm{nM}$, respectively. The other CE conditions are same as in Fig. 1.

tinguish the bound aptamer from the unbound aptamer. It is also possible to differentiate the protein-DNA complexes with different binding stoichiometry by fluorescence polarization. Previous study demonstrated that the two complexes of human immunodeficiency virus type 1 (HIV-1) reverse transcriptase (RT) and RT 26 aptamer with stoichiometry of $1: 1$ and $1: 2$ showed significantly different fluorescence polarization value $(P=0.098$ for $1: 1$, and $P=0.039$ for $1: 2$ ) [34]. In our case, since only one complex peak was observed, the complexes of thrombin-aptamer with different binding stoichiometries should be overlapped if they are to formed, and the fluorescence polarization of the complex peak should change with the varied concentration of thrombin. As shown in Fig. 3, the $P$ values of the complex peak obtained from separate electropherograms are kept constant $(0.306 \pm 0.016$ to $0.306 \pm 0.003$ ) throughout the investigated concentration range (thrombin: $0.25 \mathrm{nM}$ to $118 \mathrm{nM}$ vs $5 \mathrm{nM}$ aptamer TA35). The $P$ value of unbound aptamer in each analysis $(P=0.186 \pm 0.06)$ is also similar to that of aptamer only $(P=0.193)$. The nearly constant $P$ values for the complex formed by the different concentrations of thrombin and constant concentration of aptamer TA35 further show that the complex of thrombin-aptamer only has a single binding stoichiometry of $1: 1$.

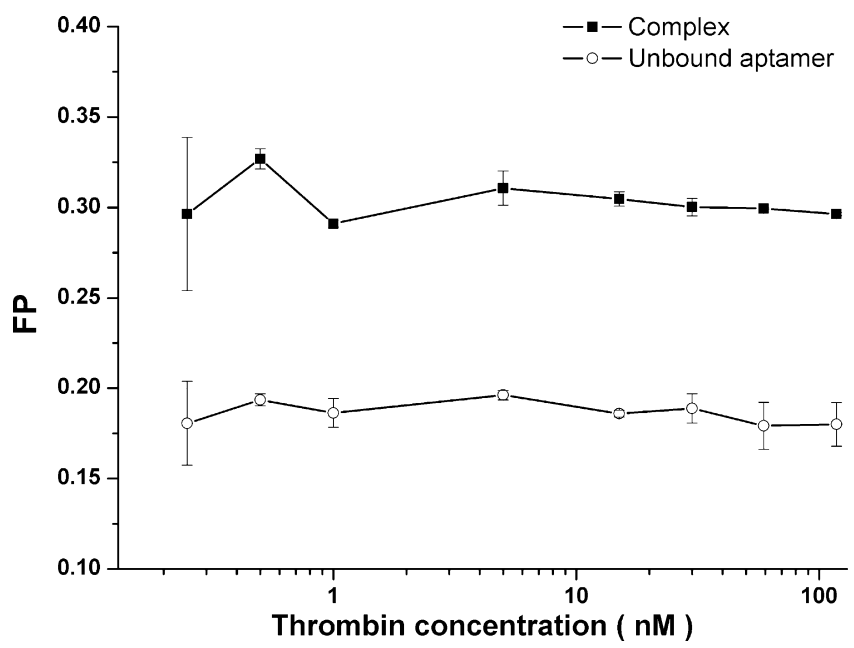

Fig. 3. The change of $P$ value obtained from the samples containing $5 \mathrm{nM}$ TA35 and varying concentration of thrombin. The $P$ values are the mean obtained from 3 measurements. The error bars were the standard deviation from the mean. 


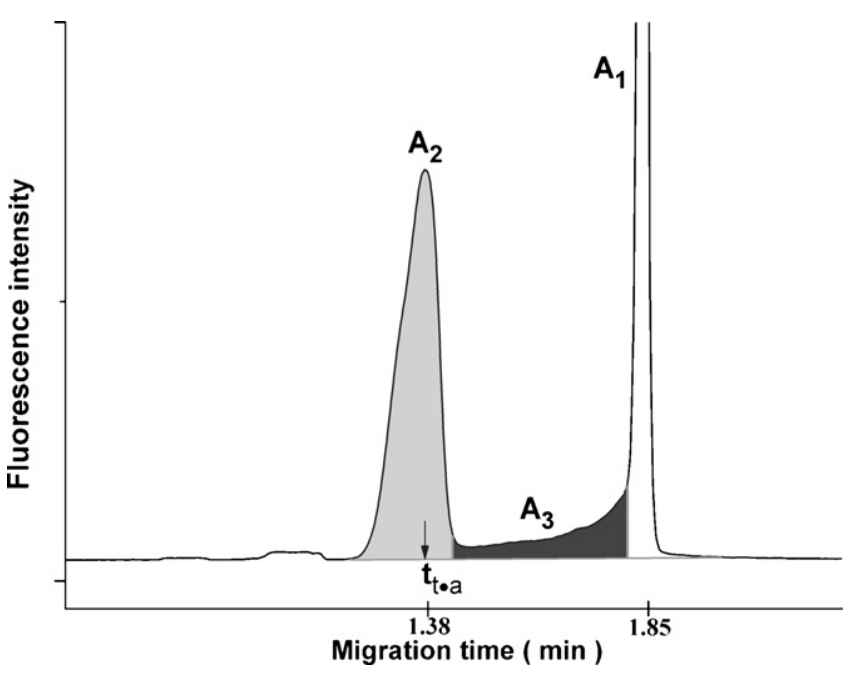

Fig. 4. Capillary electropherogram showing the dissociation of TA35 and undissociated thrombin complex. $A_{1}$ represents the peak of unbound TA35; $A_{2}$ represents the peak of the undissociated complex; $A_{3}$ represents the peak of TA35 dissociated from complex, and $t_{t \cdot a}$ is the migration time of the complex.

\subsection{Effect of metal cations}

The stability of thrombin-aptamer complex is a very important factor that determines the detection sensitivity of thrombin using aptamer as probes in APCE. The dissociation of the protein-aptamer complexes during CE separation has been observed in this work and previous work as well $[17,37]$. If the dissociation of the complex matches with the scale of the separation, a unique dissociation plateau can be formed. As shown in Fig. 4, a dissociation plateau (peak 3) was indeed observed, which is located between unbound aptamer (peak 1) and the complex (peak 2). In order to evaluate the binding of the aptamer to thrombin (A-T), the binding degree (A-T\%) was calculated from the peak area of three peaks:

Total aptamer $=$ unbound aptamer $\left(A_{1}\right)+\operatorname{complex}\left(A_{2}\right)$

$$
+ \text { dissociated aptamer }\left(A_{3}\right)
$$

$\mathrm{A}-\mathrm{T} \%=\frac{A_{2}+A_{3}}{A_{1}+A_{2}+A_{3}}$

The aptamers selected for thrombin may adopt the G-quartet structure $[28,38,39]$. The G-quartet structure can also be derived from their sequence. Previous studies showed that some metal ions could stabilize the G-quartet structure and enhanced the binding of the aptamers to thrombin [29]. For example, $\mathrm{K}^{+}$was able to form stable intramolecular cation-aptamer complexes, while $\mathrm{Mg}^{2+}$ only formed weak complex for 15-mer aptamer [29]. Here we evaluated the $\mathrm{A}-\mathrm{T} \%$ in the presence of metal ions, $\mathrm{K}^{+}$and $\mathrm{Mg}^{2+}$. Fig. 5 shows the change of the A-T\% of TA29 and TA35 bound to thrombin with increasing concentration of metal ions. In the absence of $\mathrm{K}^{+}$and $\mathrm{Mg}^{2+}$ in sample buffers, the maximum A-T\% values were achieved with a value of $67 \%$ and $74 \%$ for TA29 and TA35, respectively. The A-T\% values decreased with increasing concentration of $\mathrm{K}^{+}$or $\mathrm{Mg}^{2+}$. Therefore, it is preferable not to use $\mathrm{K}^{+}$or $\mathrm{Mg}^{2+}$ in the reaction of thrombin and aptamer TA35/TA29 for achieving maximum sensitivity in this aptamer based APCE analysis of thrombin.

The results also indicate that the A-T\% of TA29 and TA35 bound to thrombin was affected seriously by the type and concentration of metal ions. Our results are not in agreement with a recent report that $\mathrm{K}^{+}$cation has no significant influence on 29-mer-thrombin complex peak and separation performance [18]. The reasons for this negative effect of metal ions on the complex formation are not clear. However, two possibilities may be considered. The first one

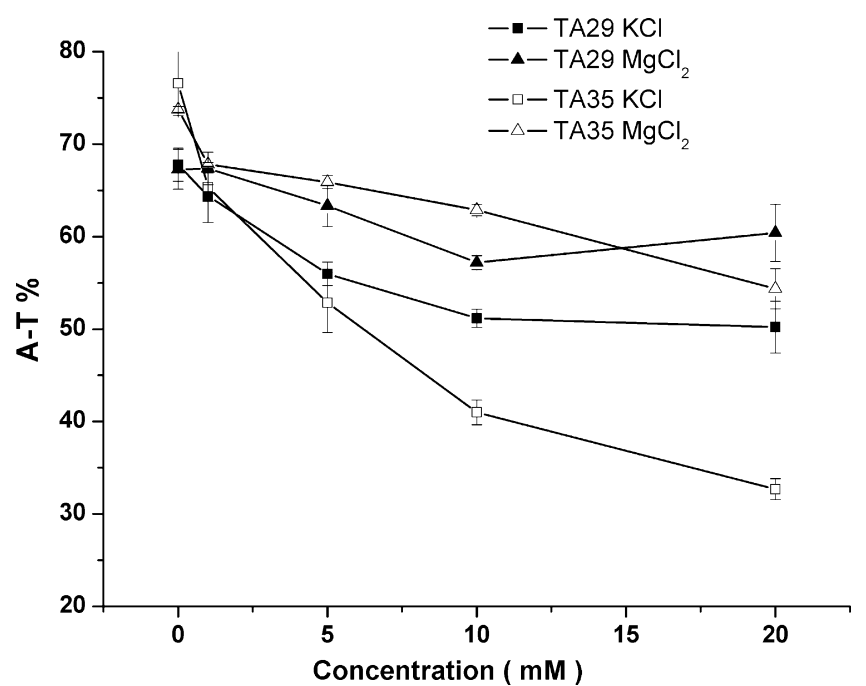

Fig. 5. Effect of metal cations on the complex formation (A-T\%) in CE/LIFP analysis The concentrations of thrombin, TA35, and TA29 are corresponding to 59, 5, $5 \mathrm{nM}$. The sample buffer is $1 \times$ TG buffer containing different concentrations of $\mathrm{KCl}$ or $\mathrm{MgCl}_{2}$. The separation buffer is $1 \times$ TG buffer.

is the accelerated dissociation kinetics of the thrombin-aptamer complex by metal ions. The second possibility is reduced ability of the aptamers to associate with thrombin by metal ions $\left(\mathrm{K}^{+}\right.$or $\left.\mathrm{Mg}^{2+}\right)$. To judge which factor is responsible for the deleterious effect of the metal cations on the complex of thrombin and aptamer, we evaluated the dissociation constants $\left(k_{d}\right)$ and dissociation rate constants $\left(k_{\text {off }}\right)$. Since the APCE/LIF analysis provided the information of undissociated complex, dissociated complex and unbound aptamer (as Fig. 4), the $k_{d}$ and $k_{\text {off }}$ values were calculated as per following formula [40]:

$k_{d}=\frac{[P]_{0}\left(1+A_{1} /\left(A_{2}+A_{3}\right)\right)-[\mathrm{DNA}]_{0}}{1+\left(A_{2}+A_{3}\right) / A_{1}}$

and

$k_{\text {off }}=\ln \left(\frac{\mathrm{A} 2+\mathrm{A} 3)}{\mathrm{A} 2}\right) / t_{t} \cdot a$

Here $A_{1}$ is the area of the peak corresponding to unbound aptamer; $A_{2}$ is the area of the peak corresponding to undissociated thrombin-aptamer complex; $A_{3}$ is the area of the peak of the exponential smear left by aptamer dissociated from the thrombin-aptamer complex during CE separation; $t_{t \cdot a}$ is the migration time of the complex. $[\mathrm{P}]_{0}$ and $[\mathrm{DNA}]_{0}$ are the total concentrations of thrombin and aptamers in the mixture, respectively. The $k_{d}$ and $k_{\text {off }}$ values of TA29 and TA35 with or without $\mathrm{K}^{+}$are calculated (Table 1 ). The $k_{d}$ values are decreased with increasing concentration of $\mathrm{KCl}$ for both TA29 and TA35, while the $k_{\text {off }}$ values display no significant changes. The results indicate that the presence of $\mathrm{K}^{+}$cannot enhance the binding of the aptamer to thrombin. On the contrary, it has an adverse impact on the binding affinity of aptamer by reducing the complex formation of thrombin-aptamer.

Table 1

Effect of $\mathrm{KCl}$ on $k_{d}$ and $k_{\text {off }}$ values of TA29 and TA35.

\begin{tabular}{cllccc}
\hline KCl concentration & \multicolumn{2}{l}{ TA29 } & & \multicolumn{2}{l}{ TA35 } \\
\cline { 2 - 3 } \cline { 5 - 6 } & $k_{d}(\mathrm{nM})$ & $k_{\text {off }}\left(\mathrm{s}^{-1}\right)$ & & $k_{d}(\mathrm{nM})$ & $k_{\text {off }}\left(\mathrm{s}^{-1}\right)$ \\
\hline $0 \mathrm{mM}$ & 21.9 & 0.036 & 14.3 & 0.030 \\
$1 \mathrm{mM}$ & 24.7 & 0.037 & 22.5 & 0.029 \\
$5 \mathrm{mM}$ & 31.1 & 0.028 & 38.4 & 0.028 \\
$10 \mathrm{mM}$ & 38.0 & 0.032 & 57.3 & 0.022 \\
$20 \mathrm{mM}$ & 46.4 & 0.041 & 102.9 & 0.036 \\
\hline
\end{tabular}




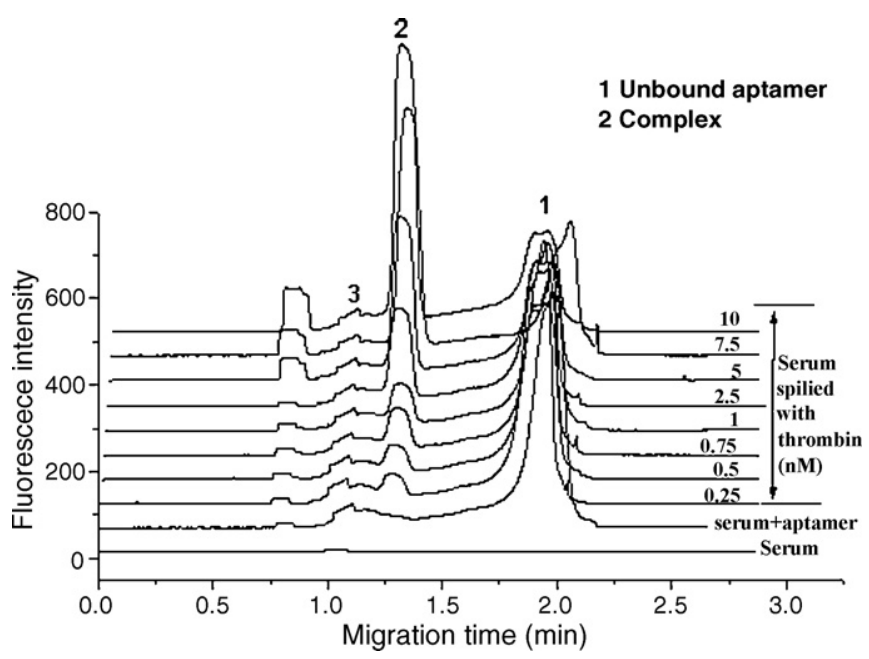

Fig. 6. Determination of thrombin in spiked serum by CE/LIFP analysis. Aptamer TA35 of $10 \mathrm{nM}$ is used as fluorescent and affinity probe. Peak 1 represents the unbound aptamer; peak 2 represents the complex; peak 3 represents new peak formation in serum by addition of aptamer. The concentrations of spiked thrombin are from 0 to $10 \mathrm{nM}$. The assay is performed using $1 \times$ TG buffer as sample and separation buffer.

According to the calculated binding constants, both aptamer TA29 and TA35 could be used for quantitative analysis of thrombin. However, the aptamer TA35 displays a lower $k_{d}$ than TA29 in the absence of metal cations. Thus, aptamer TA35 was chosen as probe for thrombin analysis.

\subsection{Quantification of thrombin}

A calibration curve for thrombin using standard thrombin solution was constructed in terms of the peak area of the complex vs the concentration of thrombin. The concentration of the aptamer TA35 was kept as $10 \mathrm{nM}$. In an expanded region of thrombin (0.06-10 nM), a linear correlation was obtained as $y=830.7 x-180.8$ with a correlation coefficient of $0.995\left(r^{2}\right)$. The LOD, taken as the lowest thrombin concentration at the threefold $S / N$, was determined to be $4.38 \times 10^{-19} \mathrm{~mol}$ in mass and $83 \mathrm{pM}$ in concentration.

To examine whether other proteins affect the assay for thrombin, we examined an abundant protein BSA on the binding of aptamer TA35 to thrombin. In this case, thrombin of $10 \mathrm{nM}$ was mixed with or without $2 \mu \mathrm{M}$ BSA in the same buffered solution containing $5 \mathrm{nM}$ aptamer TA35. The observed migration ( $1.16 \mathrm{~min})$, peak area (3623), and FP value (0.289) are consistent with those measured with thrombin alone ( $1.15 \mathrm{~min}, 3908$, and 0.278$)$. The results indicate that the binding between thrombin and aptamer is not affected by 200 -fold excess of BSA.

Human serum, diluted 50 times, was tested alone and by spiking with thrombin (in the concentration range of $0-50 \mathrm{nM}$ ). The electropherograms from which the calibration curve was constructed are shown in Fig. 6. In the absence of spiked thrombin, a peak (peak 3) at 1.07 min was observed from the control serum diluted by the electrophoresis buffer when aptamer TA35 was involved as the probe. An additional aptamer-protein complex (peak 2) at 1.29 min was observed from thrombin-spiked serum. The peak area of the complex (peak 2) increases with the increasing thrombin concentration, but that of peak 3 does not change. Probably the formation of peak 3 is due to nonspecific interaction between serum component and TA35. It has reported that the use of excess nonspecific oligonucleotides might minimize the nonspecific interactions [17]. In our study, the formation of the thrombin-aptamer complex was not affected by peak 3 , so we did not use nonspecific oligonucleotide to reduce the nonspecific interaction. In the examined range of throm- bin $(0.25-10 \mathrm{nM})$ in spiked serum, a linear correlation was obtained as $y=124.1 x+53.3$ with an $r^{2}$ of 0.991 , and LOD was $2.94 \times 10^{-19} \mathrm{~mol}$ in mass and $55.6 \mathrm{pM}$ in concentration $(S / N=3)$. A slight improvement in LOD for spiked serum was observed compared with the standard solution. This may be due to the reason that the nonspecific proteins in serum can enhance the formation and stability of thrombin-aptamer complex [41]. The RSDs $(n=3)$ along the whole concentration range were $<10 \%$ for both assays. Both in standard solution and spiked serum, the obtained LODs are much lower than that the previously reported values of $0.5 \mathrm{nM}$ (sandwich assay) [24], $9.8 \mathrm{nM}$ (APCE/LIF assay) [29], and $2 \mathrm{nM}$ (APCE/LIF assay) [18] based on thrombin aptamers.

\section{Conclusion}

In this work, we demonstrate the use of APCE/LIFP for detection and quantification of thrombin, an important protein involved in the blood coagulation, using aptamers as affinity probes. In APCE/LIFP assays, we can obtain more information on binding interactions between aptamer and thrombin during CE process through measuring the mobility and fluorescence polarization of complex simultaneously. The mobility and polarization can be used to monitor and differentiate the complexes during CE separation together. The advantage of monitoring and differentiating the formation of affinity complex using APCE/LIFP is applicable to identify target molecular on the basis of fluorescence polarization even in a complex matrix.

In this report, we successfully detected thrombin in standard solution and spiked human serum with a low LOD at sub nanomoles/liter. At the same time, we found that increasing metal ions concentration could improve separation resolution, but decrease the binding degree. Regarding the advantages of high efficient and rapid separation, low sample consumption, and high sensitivity, APCE/LIFP is a potential and powerful alternative to conventional immunoaffinity assays in clinical diagnostics.

\section{Acknowledgements}

This work is supported by the grants from the National Natural Science Foundation of China (Nos. 20707034, 20677066 and 20621703), the National Basic Research Program of China (973 program, No. 2007CB407305), and the Chinese Academy of Sciences (YZ200749) to H.W. and M.S.

\section{References}

[1] C.A. Holland, A.T. Henry, H.C. Whinna, F.C. Church, FEBS Lett. 484 (2000) 87.

[2] D. Gailani, G.J. Broze Jr., Science 253 (1991) 909.

[3] C.T. Esmon, Chest 124 (2003) 26.

[4] R. De Cristofaro, E. De Candia, J. Thromb. Thrombol. 15 (2003) 151.

[5] T. Arai, J. Miklossy, A. Klegeris, J.P. Guo, P.L. McGeer, J. Neuropathol. Exp. Neurol. 65 (2006) 19

[6] P.W. Serruys, P. Vranckx, K. Allikmets, Int. J. Clin. Pract. 60 (2006) 344.

[7] D.J. Cada, T. Levien, D.E. Baker, Hospital Pharm. 43 (2008) 577.

[8] J. Bichler, J.A. Heit, W.G. Owen, Thromb. Res. 84 (1996) 289.

[9] M. Giansily-Blaizot, R. Al Dieri, J.F. Schved, Pathophysiol. Haemost. Thromb. 33 (2003) 36.

[10] A. Schwienhorst, Cell Mol. Life Sci. 63 (2006) 2773.

[11] M.A. Shuman, P.W. Majerus, J. Clin. Invest. 58 (1976) 1249.

[12] K.E. Brummel-Ziedins, C.Y. Vossen, S. Butenas, K.G. Mann, F.R. Rosendaal, J. Thromb. Haemost. 3 (2005) 2497.

[13] R.G. Macfarlane, R. Biggs, J. Clin. Pathol. 6 (1953) 3.

[14] A. Pathak, R. Zhao, D.M. Monroe, H.R. Roberts, B.C. Sheridan, C.H. Selzman, G.A. Stouffer, J. Thromb. Haemost. 4 (2006) 60.

[15] R. Luddington, T. Baglin, J. Thromb. Haemost. 2 (2004) 1954.

[16] M.D. Rand, J.B. Lock, C. van't Veer, D.P. Gaffney, K.G. Mann, Blood 88 (1996) 3432.

[17] A. Obubuafo, S. Balamurugan, H. Shadpour, D. Spivak, R.L. McCarley, S.A. Soper, Electrophoresis 29 (2008) 3436.

[18] Y. Li, L. Guo, F. Zhang, Z. Zhang, J. Tang, J. Xie, Electrophoresis 29 (2008) 2570

[19] I. German, D.D. Buchanan, R.T. Kennedy, Anal. Chem. 70 (1998) 4540.

[20] A.D. Ellington, J.W. Szostak, Nature 346 (1990) 818.

[21] C. Tuerk, L. Gold, Science 249 (1990) 505. 
[22] X. Fang Y. Mi, J.J. Li, T. Beck, S. Schuster, W. Tan, Cell Biochem. Biophys. 37 (2002) 71.

[23] S. Fredriksson, M. Gullberg, J. Jarvius, C. Olsson, K. Pietras, S.M. Gústafsdóttir, A. Ostman, U. Landegren, Nat. Biotechnol. 20 (2002) 473.

[24] S. Centi, S. Tombelli, M. Minunni, M. Mascini, Anal. Chem. 79 (2007) 1466.

[25] S.D. Jayasena, Clin. Chem. 45 (1999) 1628.

[26] L.C. Bock, L.C. Griffin, J.A. Latham, E.H. Vermaas, J.J. Toole, Nature 355 (1992) 564.

[27] K.Y. Wang, S.H. Krawczyk, N. Bischofberger, S. Swaminathan, P.H. Bolton, Biochemistry 32 (1993) 11285.

[28] D.M. Tasset, M.F. Kubik, W. Steiner, J. Mol. Biol. 272 (1997) 688

[29] C.C. Huang, Z. Cao, H.T. Chang, W. Tan, Anal. Chem. 76 (2004) 6973.

[30] F. Perrin, J. Phys. Radium 7 (1926) 390.

[31] P. Yang, R.J. Whelan, E.E. Jameson, J.H. Kurzer, L.S. Argetsinger, C. Carter-Su, A. Kabir, A. Malik, R.T. Kennedy, Anal. Chem. 77 (2005) 2482.
[32] Q.H. Wan, X.C. Le, Anal. Chem. 71 (1999) 4183.

[33] Q.H. Wan, X.C. Le, Anal. Chem. 72 (2000) 5583.

[34] H. Fu, J.W. Guthrie, X.C. Le, Electrophoresis 27 (2006) 433

[35] M. Gong, I. Nikcevic, K.R. Wehmeyer, P.A. Limbach, W.R. Heineman, Electrophoresis 29 (2008) 1415

[36] X.C. Le, Q.H. Wan, M.T. Lam, Electrophoresis 23 (2002) 903.

[37] D.D. Buchanan, E.E. Jameson, J. Perlette, A. Malik, R.T. Kennedy, Electrophoresis $24(2003) 1375$.

[38] K.Y. Wang, S. McCurdy, R.G. Shea, S. Swaminathan, P.H. Bolton, Biochemistry 32 (1993) 1899.

[39] H.A. Ho, M. Leclerc, J. Am. Chem. Soc. 126 (2004) 1384.

[40] S.N. Krylov, Electrophoresis 28 (2007) 69

[41] H.L. Wang, M.L. Lu, M. Weinfield, X.C. Le, Anal. Chem. 75 (2003) 247 